\title{
THE STABILITY OF VECTOR RENEWAL EQUATIONS PERTAINING TO HETEROGENEOUS CHEMICAL REACTION SYSTEMS*
}

\author{
By
}

\author{
A. E. DEGANCE** ANd L. E. JOHNS \\ University of Florida, Gainesville
}

\begin{abstract}
For a class of vector renewal equations arising in the theory of spatially nonuniform chemical reaction processes, stability conditions are given in terms of the physicochemical operators $\mathbf{D}$ and $\mathbf{K}$. In particular, we provide conditions for the stability of an initially uniform, multicomponent film bounding a planar catalytic surface, and for the asymptotic stability of a reaction system composed of a population of catalyst particles. We obtain such results by identifying a sup norm which is naturally induced from the factors that symmetrize $\mathbf{D}$ and/or $\mathbf{K}$. Further, we exhibit conditions under which a special class of vector renewal equations has positive solutions.
\end{abstract}

Notation.

$$
\begin{aligned}
& \mathbf{x}=\left(\begin{array}{c}
x_{1} \\
x_{2} \\
\vdots
\end{array}\right) ; \quad \mathbf{x}^{+}=\left(x_{1}, x_{2}, \cdots\right) \\
& \mathbf{V}=\left(\mathbf{v}_{1}, \mathbf{v}_{2}, \cdots\right), \mathbf{v}_{i}=\left(\begin{array}{c}
v_{1 i} \\
v_{2 i} \\
\vdots
\end{array}\right) ; \quad \mathbf{V}^{+}=\left(\begin{array}{c}
\mathbf{v}_{1}^{+} \\
\mathbf{v}_{2}^{+} \\
\vdots
\end{array}\right)
\end{aligned}
$$

$\operatorname{diag}(\mathbf{V})=\operatorname{diag}\left(v_{11}, v_{22}, \cdots\right)$.

$\Phi=\left\{\mathbf{V}: v_{i i} \geq 0, i \neq j\right\}$, set of input-output matrices.

$\Phi_{0}=\left\{\mathrm{V} \in \Phi: \sum_{i} v_{i i}=0\right\}$, set of input-output matrices whose column sums vanish.

$P=\left\{\mathrm{V}: v_{i i} \geq 0\right\}$, set of positive matrices.

$M=\left\{\mathrm{V} \in P: \sum_{i} v_{i j}=1\right\}$, set of Markoff matrices.

$R^{n}=$ space of $n \times 1$ vectors.

$L\left(R^{n}\right)=$ space of $n \times n$ matrices.

$C[0, \infty]=$ space of continuous functions on $[0, \infty]$, viz. $b \in C[0, \infty]$ iff $b \in C[0, \infty)$, $\lim _{t \rightarrow \infty} b(t)=b(\infty)<\infty$.

1. Introduction. The theory of functional differential equations has been used to provide insight into the physics of mixing processes by Driver [5] and by Johns and

* Received June 16, 1974; revised version received October 22, 1974.

** Present address: Systems Control, Inc., Palo Alto, Calif., 94304. 
Reed [10]. Further, certain problems arising in investigations of the mixing - diffusionchemical reaction sequence in particulate suspensions can be formulated as problems in integral equations which reflect either the role of spatial gradients, at the local or single particle level, or the role of finite (non-infinite) renewal probabilities, at the global or collective particle level. Each factor is characteristic of imperfect contacting and each must be understood if the structure of systems departing from the idealization of perfect contacting, i.e. spatial uniformity, is to be understood.

In particular, the chemical response of a thin, initially uniform, layer bounding a planar catalytic surface is given by

$$
\mathbf{y}(t)=\mathbf{y}(0)+\int_{0}^{l} f_{0}\left(l^{-2} \mathbf{D} \tau\right) l^{-1} \mathbf{K} \mathbf{y}(t-\tau) d \tau
$$

wherein $f_{0}(x)=2 \sum_{n=1}{ }^{\infty} \exp \left(-\alpha_{n}{ }^{2} x\right), \alpha_{n}=\frac{1}{2} \pi(2 n-1)$ and $\exp \left(-\alpha_{n}{ }^{2} l^{-2} \mathbf{D}_{\tau}\right)$ has its usual meaning (cf. [1]), and where $l, \mathbf{D}, \mathbf{K}$ are the physicochemical parameters expressed on the length scale of $\left(a n_{T}\right)^{-1}$. The vector $\mathbf{y}(t) \in R^{(N-1)}$ gives the limiting composition of the film-like region on approaching the catalytic plane, $z=0$; the distribution throughout the film may be found from

$$
\mathbf{x}(z, t)=\mathbf{y}(0)+\int_{0}^{t} f\left(l^{-2} \mathbf{D} \tau, z\right) l^{-1} \mathbf{K} \mathbf{y}(t-\tau) d \tau
$$

where $f(x, z)=2 \sum_{n=1}{ }^{\infty} \cos \left(\alpha_{n} z\right) \exp \left(-\alpha_{n}{ }^{2} x\right)$ and $\mathbf{x}(0, t)=\mathbf{y}(t)$.

If the thin film is replaced by an initially uniform reservoir of infinite extent, Eq. (1) becomes (on letting $l \rightarrow \infty$ )

$$
\mathbf{y}(t)=\mathbf{y}(0)+\int_{0}^{t} \frac{1}{(\pi \tau)^{1 / 2}} \mathbf{D}^{-1 / 2} \mathbf{K} \mathbf{y}(t-\tau) d \tau
$$

which is comparatively well understood (cf. [9]) and which provides the only basis extant for investigating the chemical dynamics of a collection of catalyst particles under conditions of imperfect contacting. In particular, if mixing is random, i.e. if particles are indistinguishable for renewal purposes, the batchwise performance of slurry systems in which hydrogenations, polymerizations, etc. take place proceeds according to

$$
\mathbf{y}(t)=\mathbf{y}(0)+\int_{0}^{t}(\mathbf{A}+\mathbf{B}(\tau)) \mathbf{y}(t-\tau) d \tau
$$

which follows from

$$
\frac{d}{d t} \mathbf{y}=\int_{0}^{t} p \exp (-p \tau) \mathbf{K} \mathbf{T}\left(-\mathbf{D}^{-1 / 2} \mathbf{K} \sqrt{ } \tau\right) \mathbf{y}(t-\tau) d \tau
$$

given in [4], where $p>0, T(x)=\exp \left(x^{2}\right) \operatorname{erfc}(x)$,

$$
\begin{aligned}
\mathbf{A}= & \mathbf{K}\left(\mathbf{I}-p^{-1 / 2} \mathbf{D}^{-1 / 2} \mathbf{K}\right)^{-1}=-\mathbf{B}(0) \\
\mathbf{B}(\tau)= & -\exp (-p \tau) \mathbf{K}\left(\mathbf{I}-\left(p^{-1 / 2} \mathbf{D}^{-1 / 2} \mathbf{K}\right)^{2}\right)^{-1} \\
& \times\left(T\left(-\mathbf{D}^{-1 / 2} \mathbf{K} \sqrt{ } \tau\right)+T\left((p \tau)^{1 / 2}\right) p^{-1 / 2} \mathbf{D}^{-1 / 2} \mathbf{K}\right)
\end{aligned}
$$

and where if re $\left(\Lambda_{i}\right)<0, \Lambda_{i}$ an eigenvalue of $\mathrm{D}^{-1 / 2} \mathrm{~K}$,

$$
\mathbf{B}(t)=-\int_{t}^{\infty} p \exp (-p \tau) \mathbf{K} T\left(-\mathbf{D}^{-1 / 2} \mathbf{K} \sqrt{ } \tau\right) d \tau .
$$

The vector $y$ represents the chemical state of the suspending medium. 
The scalar $p$ characterizes transport, via macroscopic fluctuations, from the suspending medium to the neighborhood of the particles whereas the matrices $\mathbf{D}$ and $\mathbf{K}$ characterize transport, via molecular diffusion, in the boundary layers bordering the active surface and the chemical processes taking place thereon, respectively. Here physicochemical coupling in multicomponent systems is of interest; thus we presume that the chemical networks are representible by linear chemical rate laws and investigate problems originating in the vector character of Eqs. (1), (2), and (3).

In all of the foregoing, $N(\geq 3)$ component systems are understood and $\mathbf{y}$, a $n$-vector where $n$ may be $r, N-1, N$, reflects a displacement from either a local or a global rest state. In each section of the paper there is a preferred state space which simplifies the investigation carried out therein. Thus, we introduce the spaces $R^{n}, n=r, N-1$, $N$ but, because there is little physical content in the selection of $R^{n}$, we do not provide distinctive symbols for the parameters of the problems formulated therein, preferring to repeat $\mathbf{y}, \mathbf{D}$, and $\mathbf{K}$. At the beginning of each section, however, we give the conditions on $\mathbf{D}$ and $\mathbf{K}$ used thereinafter insofar as they deviate from the minimum conditions of physical admissibility. These latter conditions once established in a preferred or natural state space carry over to all others. In particular, the diffusive and chemical processes, individually, will be presumed physically well behaved; thus $\mathbf{D}$ and $\mathbf{K}$ will be presumed real and each will have a complete set of eigenvectors, the eigenvalues of $\mathbf{D}$ being real and positive, the eigenvalues of $\mathbf{K}$ being real and negative. Alternatively, $-\mathbf{D}$ and $\mathbf{K}$ being symmetrizable as well as stable, we can assert that the sets $G_{-D}$ and $S_{\kappa}$ where

$$
\mathcal{G}_{-D}=\left\{\mathbf{G}^{+}=\mathbf{G}>0: \mathbf{G}(-\mathbf{D})=(\mathbf{G}(-\mathrm{D}))^{+}<0\right\}
$$

and

$$
S_{K}=\left\{\mathbf{G}^{+}=\mathbf{G}>0: \mathbf{G K}=(\mathbf{G K})^{+}<0\right\}
$$

are not empty. In systems which are physically and chemically simple, it is evident from above that $\mathbf{\Lambda}=\mathbf{D}^{-1 / 2} \mathbf{K}$ and $\mathbf{A}=\mathbf{K}\left(\mathbf{I}-p^{-1 / 2} \mathbf{D}^{-1 / 2} \mathbf{K}\right)^{-1}$ are important determinants of system evolution on the local and the global scale, respectively. Moreover, for $N=3$ it is easy to exhibit physically admissible $\mathbf{D}$ and $\mathbf{K}$ such that either $\mathbf{D}^{-1 / 2} \mathbf{K}$ or $\mathbf{K}(\mathbf{I}-$ $\left.p^{-1 / 2} \mathbf{D}^{-1 / 2} \mathbf{K}\right)^{-1}$ have nonreal eigenvalues; further, even if the eigenvalues of the product operators are real, under certain conditions one or more can be positive, which is sufficient for local and/or global instability.

For a linear chemical network arising either from a system of unimolecular steps or from a linearization of a system of bimolecular steps or other less common mass action kinetics, the formal solution of each of the above equations has been exhibited elsewhere (that of Eq. (1a) in [8], that of Eq. (2) in [9], and that of Eq. (3) in [4]). The predictions of the theory that have been reported verify that diffusion reaction interference in multicomponent systems is often the source of otherwise unexplained selectivity in certain complex chemical networks. In general, the response of a system depends on the latent roots of certain $\zeta$-matrices arising in the construction of the solution of Eqs. (1a), (2), and (3); in particular, it is necessary to investigate the regions of the complex plane in which the latent roots of:

Eq. (1a): $\zeta l^{-1} \mathbf{D}^{1 / 2} \cos \left(\zeta l \mathbf{D}^{-1 / 2}\right)-\sin \left(\zeta l \mathbf{D}^{-1 / 2}\right) l^{-1} \mathbf{K}$

Eq. (2): $\zeta \mathbf{I}-\mathbf{D}^{-1 / 2} \mathbf{K}$

Eq. (3): $\left(\zeta^{2}-p\right) \zeta\left(\zeta \mathbf{I}-\mathbf{D}^{-1 / 2} \mathbf{K}\right)-p \mathbf{K}$ 
lie for parametric values of $\mathbf{D}$ and $\mathbf{K}$ if the variety in the pattern of system response is to be properly classified. Although we readily express assymptotic stability conditions in the $\zeta_{i}$ :

Eq. (1a): $\operatorname{re}\left(\zeta_{i}^{2}\right)>0$

Eq. (2): $\operatorname{re}\left(\zeta_{i}\right)<0$ or $\operatorname{re}\left(\zeta_{i}{ }^{2}\right)<0$ if $\operatorname{re}\left(\zeta_{i}\right) \geq 0$

Eq. (3): $\operatorname{re}\left(\zeta_{i}\right) \leq 0$ or $\operatorname{re}\left(\zeta_{i}{ }^{2}\right)<p$ if $\operatorname{re}\left(\zeta_{i}\right)>0$,

the dependence of the $\zeta_{i}$ on the matrix parameters $\mathbf{D}$ and $\mathbf{K}$ is implicit. Thus, the formal solutions of Eqs. (1a), (2), and (3) are not of much use in classifying the consequences of physicochemical interactions and investigations of the determinantal equations for the $\zeta_{i}$ must be supplemented, possibly by direct investigations of the renewal equations using tools ordinarily reserved for nonlinear equations.

We give the conclusions of such a study herein where the basis of much of our work is the comparison theorem of Nohel [12]. However, inasmuch as Eqs. (1a), (2), and (3) are linear integral equations, we require only the following special result, which can be proved directly by construction:

Lemma A. Let $a(t) \geq 0$ and $b(t) \geq 0, a, b \in \mathfrak{R}[0, T] T>0$; then if

$$
x(t) \leq b(t)+\int_{0}^{t} a(t-\tau) x(\tau) d \tau
$$

we have that $x(t) \leq \hat{x}(t)$ where

$$
\hat{x}(t)=b(t)+\int_{0}^{t} a(t-\tau) \hat{x}(\tau) d \tau .
$$

Moreover, if $\int_{0}^{\infty} a(\tau) d \tau<1$ and $b \in C[0, \infty]$ we have that $0 \in C[\hat{x}, \infty]$ where

$$
\hat{x}(\infty)=b(\infty)\left(1-\int_{0}^{\infty} a(\tau) d \tau\right)^{-1} .
$$

In Sec. 2 we investigate Eq. (1) and conclude that if $l<l_{0}=D_{\min } /\|\mathbf{K}\|$ then $\mathbf{y}(t)$ is bounded.

In Sec. 3 we investigate Eq. (3) and give sufficient conditions for its asymptotic stability; we also show that under such conditions the solution of Eq. (3) can be approximated by the solution of

$$
d \mathbf{y}_{0} / d t=\mathbf{K}\left(\mathbf{I}-p^{-1 / 2} \mathbf{D}^{-1 / 2} \mathbf{K}\right)^{-1} \mathbf{y}_{0}, \quad \mathbf{y}_{0}(0)=\mathbf{y}(0) .
$$

In Sec. 4 we show that under the condition $\mathcal{G}_{-D} \cap \mathcal{G}_{K} \neq 0$, which is realized for thermodynamically ideal systems, e.g. a network of chemical isomers, the region of asymptotic stability of Eq. (3), found in Sec. 3, can be enlarged using a technique developed by Duffin [6].

In Sec. 5 we prove that $\mathbf{D}^{-1 / 2} \mathbf{K} \in \Phi_{0}$ is necessary and sufficient for

$$
T\left(-\mathrm{D}^{-1 / 2} \mathrm{~K} \sqrt{ } t\right) \in M \text {. }
$$

Further we identify a set of functions $S$ such that if $f \in S$ and $\mathbf{A} \in \Phi_{0}$ then $f(\mathbf{A}) \in M$; the extension, if $f \in S$ and $\mathbf{A} \in \Phi$ then $f(\mathbf{A}) \in P$, is immediate. Such theorems place important conditions on physically admissible products, $\mathbf{D}^{-1 / 2} \mathbf{K}$, and have special significance for systems operating in the neighborhood of the lines $x_{i}=0, i=1, \cdots, N$, which bound the state space. 
2. On the boundedness of the solution of Eq. (1). We introduce $\mathbf{M}_{0}(t)$ where

$$
\mathbf{M}_{0}(t)=\mathbf{I}+\int_{0}^{t} f_{0}\left(l^{-2} \mathbf{D} \tau\right) l^{-1} \mathbf{K} \mathbf{M}_{0}(t-\tau) d \tau
$$

thus $\mathbf{y}(t)=\mathbf{M}_{0}(t) \mathbf{y}(0)$, where $\mathbf{y}(t) \in R^{(N-1)}$, and $\mathbf{y}$ is bounded iff $\mathbf{M}_{0}$ is bounded. We introduce a norm on $L\left(R^{(N-1)}\right)$ and exhibit sufficient conditions for the existence of $m \in C[0, \infty]$ such that $m(t) \geq\left\|\mathbf{M}_{0}(t)\right\|, t \in[0, \infty)$.

We limit ourselves to norms on $L\left(R^{(N-1)}\right)$ induced from inner products on $R^{(N-1)}$, viz.

$$
\|\mathbf{V}\|=\sup _{|| \mathbf{x} \|=1}\|\mathbf{V x}\|
$$

where $\|\mathbf{x}\|=(\mathbf{x}, \mathbf{G} \mathbf{x})^{1 / 2}$ and $\mathbf{G}=\mathbf{G}^{+}>0, \mathbf{G} \in L\left(R^{N-1}\right)$. It follows that

$$
\|\mathbf{V}\|=\max \left\{\sqrt{ } V_{i}: \operatorname{det}\left(V_{i} \mathbf{I}-\mathbf{V V}^{*}\right)=0\right\}
$$

where $\mathbf{V}^{*}$ is defined via $(\mathbf{V} \mathbf{x}, \mathbf{G y})=\left(\mathbf{x}, \mathbf{G} \mathbf{V}^{*} \mathbf{y}\right.$ ) (cf. Shilov [13]), and hence $\mathbf{V}^{*}=\mathbf{G}^{-1} \mathbf{V}^{+} \mathbf{G}$.

We let $\mathbf{G}=\mathbf{G}_{D} \in \mathcal{G}_{-D}$ and find that $\left\|f_{0}\left(l^{-2} \mathbf{D} t\right)\right\|=f_{0}\left(l^{-2} D_{\min } t\right)$ where $D_{\min }=$ $\min \left\{D_{i}: \operatorname{det}\left(D_{i} \mathbf{I}-\mathbf{D}\right)=0\right\}=\left\|\mathbf{D}^{-1}\right\|^{-1}$ inasmuch as $\mathbf{G}_{D} f_{0}\left(l^{-2} \mathbf{D} t\right)=\left(\mathbf{G}_{\nu} f_{0}\left(l^{-2} \mathbf{D} t\right)\right)^{+}$ implies $f_{0}^{*}\left(l^{-2} \mathbf{D} t\right)=f_{0}\left(l^{-2} \mathbf{D} t\right)$ and therefore, $\left\|f_{0}\left(l^{-2} \mathbf{D} t\right)\right\|=\max \left\{\left|f_{0}\left(l^{-2} D_{i} t\right)\right|\right\}$. It follows, therefore, on using the product and integral inequalities for norms of operators $\mathbf{V} \in[0, t]$ (cf. Bellman [1]), that

$$
\left\|\mathbf{M}_{0}(t)\right\| \leq 1+\int_{0}^{t} f_{0}\left(l^{-2} D_{\min }(t-\tau)\right) l^{-1}\|\mathbf{K}\|\left\|\mathbf{M}_{0}(\tau)\right\| d \tau
$$

thus we find from Lemma A that

$$
\left\|\mathbf{M}_{0}(t)\right\| \leq m(t)
$$

where

$$
m(t)=1+\int_{0}^{t} f_{0}\left(l^{-2} D_{\min }(t-\tau)\right) l^{-1}\|\mathbf{K}\| m(\tau) d \tau
$$

and where, inasmuch as $f_{0}\left(l^{-2} D_{\min } t\right)>0, m(t)$ is a positive strictly increasing function. We conclude that, for all $l>0, m(t)$, and therefore $\mathbf{M}_{0}(t)$, is bounded on each finite interval $[0, T], T>0$. Further,

$$
\lim _{t \rightarrow \infty} m(t)<\infty \text { iff } \int_{0}^{\infty} f_{0}\left(l^{-2} D_{\min } \tau\right) d \tau l^{-1}\|\mathbf{K}\|\left(=l^{-1}\|\mathbf{K}\| / l^{-2}\left\|\mathbf{D}^{-1}\right\|^{-1}\right)<1 ;
$$

also if $l\left\|\mathbf{D}^{-1}\right\|\|\mathbf{K}\|<1$ then $\lim _{t \rightarrow \infty} m(t)=1 /\left(1-l\left\|\mathbf{D}^{-1}\right\|\|\mathbf{K}\|\right) \equiv m(\infty)$. Thus, if $l / l_{0}<1$, where $l_{0}=\left(\left\|\mathbf{D}^{-1}\right\|\|\mathbf{K}\|\right)^{-1}$, then $\left\|\mathbf{M}_{0}(t)\right\| \leq m(t) \leq m(\infty)<\infty$ for $t \in[0, \infty)$ and $\mathbf{y}$ is bounded. Now $\mathbf{x}(z, t)=\mathbf{M}(z, t) \mathbf{y}(0)$, where

$$
\mathbf{M}(z, t)=\mathbf{I}+\int_{0}^{t} f\left(l^{-2} \mathbf{D} \tau, z\right) l^{-1} \mathbf{K} \mathbf{M}_{0}(t-\tau) d \tau
$$

and, therefore,

$$
\|\mathbf{M}(z, t)\| \leq 1+\int_{0}^{t} f_{0}\left(l^{-2} D_{\min } \tau\right) l^{-1}\|\mathbf{K}\| m(t-\tau) d \tau=m(t)
$$

inasmuch as

$$
\left\|f\left(l^{-2} \mathrm{D} \tau, z\right)\right\| \leq f_{0}\left(l^{-2} D_{\min } \tau\right)
$$


Thus, the condition $l / l_{0}<1$ is sufficient for the boundedness of $\mathbf{x}$, and hence for each admissible pair of operators $\mathbf{D}$ and $\mathbf{K}$, we can compute a film thickness interval $\left[0, l_{0}\right]$ on which each particle of a suspension is physicochemically stable.

3. Asymptotic stability of Eq. (3). We use the reduced state variable formulation of Eq. (3) in which $\mathbf{y} \in R^{r}$, cf. [4]. Further, we presume that, in addition to the restrictions on $\mathbf{D}$ and $\mathbf{K}$ set forth in the introduction, $\mathbf{\Lambda} \equiv \mathbf{D}^{-1 / 2} \mathbf{K}$ and $\mathbf{A} \equiv \mathbf{K}\left(\mathbf{I}-p^{-1 / 2} \mathbf{D}^{-1 / 2} \mathbf{K}\right)^{-1}$ have complete sets of eigenvectors and real, strictly negative, eigenvalues $\Lambda_{i}$ and $A_{i}$ respectively. In particular, $\boldsymbol{\Lambda}$ and $\boldsymbol{A}$ are symmetrizable as well as stable and we let $S_{\Lambda}=\left\{\mathbf{G}=\mathbf{G}^{+}>0: \mathbf{G \Lambda}=(\mathbf{G} \mathbf{\Lambda})^{+}<0\right\}$ and $\mathcal{G}_{\boldsymbol{A}}=\left\{\mathbf{G}=\mathbf{G}^{+}>0: \mathbf{G A}=(\mathbf{G A})^{+}<0\right\}$.

We observe that Lemma $\mathbf{A}$ cannot be used directly inasmuch as $\mathbf{B}(t) \in \mathfrak{R}[0, \infty]$ but $A \notin \Re[0, \infty]$. Thus, we reexpress Eq. (3) in the following way:

$$
\mathbf{y}(t)=\mathbf{M}(t) \mathbf{y}(0)
$$

where

$$
\mathbf{M}(t)=\exp (\mathbf{A} t)+\int_{0}^{t}\left(\mathbf{B}(\tau)+\int_{0}^{\tau} \mathbf{A} \exp \left(\mathbf{A}\left(\tau-\tau_{0}\right)\right) \mathbf{B}\left(\tau_{0}\right) d \tau_{0}\right) \mathbf{M}(t-\tau) d \tau
$$

and where $\int_{0}^{t} \mathbf{A} \exp (\mathbf{A}(t-\tau)) \mathbf{B}(\tau) d \tau \in \mathcal{R}[0, \infty]$. We investigate the boundedness of $\mathbf{M}$ using the method of Sec. 2 .

On letting $\left\{\mathbf{y}_{i}\right\}$ be the set of right eigenvectors of $\mathbf{A}$ and the sets $\left\{\mathbf{y}_{i}\right\}$ and $\left\{\mathbf{z}_{i}\right\}$ be biorthogonal, i.e. $\left(\mathbf{z}_{i}, \mathbf{y}_{i}\right)=\delta_{i i}$, we find that $\mathbf{A}^{*}=\mathbf{A}$, where $\mathbf{A}^{*}$ is defined via $\left(\mathbf{x}, \mathbf{G}_{\mathbf{A}} \mathbf{A y}\right)$ $=\left(\mathbf{A}^{*} \mathbf{x}, \mathbf{G}_{\boldsymbol{A}} \mathbf{y}\right)$ and $\mathbf{G}_{\boldsymbol{A}}=\sum_{i=1}^{{ }^{r}} \mathbf{z}_{i} \mathbf{z}_{i}{ }^{+} \in \mathcal{G}_{\boldsymbol{A}}$, viz. $\mathbf{A}$ is selfadjoint in the inner product $\left(, \mathrm{G}_{\mathbf{A}}\right)$. Thus, we place the following norm on $L\left(R^{r}\right)$ :

$$
\|\mathbf{V}\|=\sup _{\| \mathbf{x}||=1}\|\mathbf{V x}\|
$$

where $\|\mathbf{x}\|=\left(\mathbf{x}, \mathbf{G}_{A} \mathbf{x}\right)^{1 / 2}$. The adjoints of $\mathbf{V}$ in the inner products $\left(, \mathbf{G}_{A}\right)$ and $($,$) are$ related via $\mathbf{V}^{*}=\mathbf{G}_{\boldsymbol{A}}{ }^{-1} \mathbf{V}^{+} \mathbf{G}_{\boldsymbol{A}}$.

It follows that $\|\mathbf{A}\|=\max \left\{\left|A_{i}\right|\right\}$ and that

$$
\|f(\mathbf{A})\|=\max \left\{\left|f\left(A_{i}\right)\right|\right\}
$$

and thus we find that

$$
\begin{aligned}
\|\mathbf{M}(t)\| \leq & \exp (-a t) \\
& +\int_{0}^{t}\left(\|\mathbf{B}(\tau)\|+\int_{0}^{\tau}\|\mathbf{A}\| \exp \left(-a\left(\tau-\tau_{0}\right)\right)\left\|\mathbf{B}\left(\tau_{0}\right)\right\| d \tau_{0}\right)\|\mathbf{M}(t-\tau)\| d \tau
\end{aligned}
$$

where $a=\min \left\{\left|A_{i}\right|\right\}=\left\|\mathrm{A}^{-1}\right\|^{-1}$.

Now $\|\mathbf{B}(t)\|$ turns out to be difficult to estimate, but if we can exhibit a function $b(t)$, for which $b(t) \geq\|\mathbf{B}(t)\|, t \in[0, \infty)$, then it follows that

$$
\|\mathbf{M}(t)\| \leq \exp (-a t)+\int_{0}^{t}\left(b(\tau)+\int_{0}^{\tau}\|\mathbf{A}\| \exp \left(-a\left(\tau-\tau_{0}\right)\right) b\left(\tau_{0}\right) d \tau_{0}\right)\|\mathbf{M}(t-\tau)\| d \tau
$$

and we can conclude that

$$
\|\mathbf{M}(t)\| \leq m(t)
$$


where

$m(t)=\exp (-a t)+\int_{0}^{t}\left(b(\tau)+\int_{0}^{\tau}\|\mathbf{A}\| \exp \left(-a\left(\tau-\tau_{0}\right)\right) b\left(\tau_{0}\right) d \tau_{0}\right) m(t-\tau) d \tau$.

Further, if

$$
\lim _{t \rightarrow \infty} \int_{0}^{t}\left(b(\tau)+\int_{0}^{t} \int_{0}^{\tau}\|\mathbf{A}\| \exp \left(-a\left(\tau-\tau_{0}\right)\right) b\left(\tau_{0}\right) d \tau_{0}\right) d \tau<1
$$

or, what is equivalent, if

$$
g \equiv\left(1+\|\mathbf{A}\|\left\|\mathbf{A}^{-1}\right\|\right) \int_{0}^{\infty} b(\tau) d \tau<1,
$$

we find that

$$
\lim _{t \rightarrow \infty} m(t)=\lim _{t \rightarrow \infty} \frac{\exp (-a t)}{1-g}=0
$$

and we conclude that

$$
\lim _{t \rightarrow \infty} \mathbf{M}(t)=\mathbf{0}
$$

We observe that $\mathbf{M}_{0}(t) \equiv \exp (\mathbf{A} t)$ is the zeroth Picard iterate of Eq. (4); it follows that

$$
\left\|\mathbf{M}(t)-\mathbf{M}_{0}(t)\right\| \leq m(t)-m_{0}(t)
$$

where $m_{0}(t) \equiv \exp (a t)$ is the zeroth Picard iterate of Eq. (5). Further, if $g<1$ then $m(t)-m_{0}(t)<g /(1-g)$. Estimates of $\left\|\mathbf{M}(t)-\mathbf{M}_{0}(t)\right\|$ are important insofar as $\mathbf{M}_{\mathbf{0}}$ permits a simple characterization of the roles of $p, \mathbf{D}$ and $\mathbf{K}$ which leads to the identification of chemically and physically controlled regions of operation.

We now exhibit a function $b(t)$ which majorizes $\|\mathbf{B}(t)\|$ and in so doing transforms the inequality $g<1$ into a concrete condition on $p, \mathrm{D}^{-1 / 2}$ and $\mathrm{K}$ sufficient for the asymptotic stability of Eq. (3). Using

$$
\mathbf{B}(t)=-\int_{t}^{\infty} p \exp (-p \tau) \mathbf{K} T(-\mathbf{\Lambda} \sqrt{ } \tau) d \tau
$$

we find

$$
\|\mathbf{B}(t)\| \leq \int_{\imath}^{\infty} p \exp (-p \tau)\|\mathbf{K}\|\|T(-\mathbf{\Lambda} \sqrt{ } \tau)\| d \tau
$$

however, $\|T(-\Lambda \sqrt{ } t)\|$ is difficult to estimate inasmuch as \|\| derives from $\mathbf{G}_{\boldsymbol{A}} \in \mathcal{G}_{\boldsymbol{A}}$, not from $\mathbf{G}_{\Lambda} \in S_{\Lambda}$, and, in general, $S_{\Lambda} \cap S_{\boldsymbol{A}}=\varnothing$. We let $S_{\Lambda}{ }^{A} \equiv \sup \left(\mathbf{x}, \mathbf{G}_{\boldsymbol{A}} \mathbf{x}\right) /\left(\mathbf{x}, \mathbf{G}_{\Lambda} \mathbf{x}\right)$, where $G_{\Lambda} \in G_{\Lambda}$ and $S_{\Lambda}{ }^{A}$ can be evaluated via

$$
\begin{aligned}
S_{\Lambda}{ }^{A} & =\sup _{(\mathbf{x}, \mathbf{G} \mathbf{x})=1}\left(\mathbf{x}, \mathbf{G}_{A} \mathbf{x}\right)=\sup _{(\mathbf{y}, \mathbf{y})=1}\left(\mathbf{y}, \mathrm{G}_{\Lambda}{ }^{-1 / 2} \mathrm{G}_{A} \mathrm{G}_{\Lambda}{ }^{-1 / 2} \mathbf{y}\right) \\
& =\max \left\{a_{i}: \operatorname{det}\left(a_{i} \mathbf{I}-\mathrm{G}_{\Lambda}^{-1 / 2} \mathrm{G}_{A} \mathrm{G}_{\Lambda}^{-1 / 2}\right)=0\right\},
\end{aligned}
$$

and find that

$$
\left(\mathbf{x}, \mathbf{G}_{A} \mathbf{x}\right) \leq S_{\Lambda}{ }^{A}\left(\mathbf{x}, \mathbf{G}_{\Lambda} \mathbf{x}\right)
$$


We conclude, therefore, that

$$
\begin{aligned}
\|T(-\boldsymbol{\Lambda} \sqrt{ } t)\| & =\sup _{\left(\mathbf{x}, \mathbf{G}_{\mathbf{A} \mathbf{x})=1}\right.}\left(T(-\boldsymbol{\Lambda} \sqrt{ } t) \mathbf{x}, \mathrm{G}_{A} T(-\boldsymbol{\Lambda} \sqrt{ } t) \mathbf{x}\right)^{1 / 2} \\
& \leq\left(S_{\Lambda}{ }^{A} S_{A}{ }^{\Lambda}\right)^{1 / 2} \sup _{\left(\mathbf{x}, G_{\Lambda} \mathbf{x}\right)=1}\left(T(-\boldsymbol{\Lambda} \sqrt{ } t) \mathbf{x}, \mathbf{G}_{\Lambda} T(-\boldsymbol{\Lambda} \sqrt{ } t) \mathbf{x}\right)^{1 / 2} .
\end{aligned}
$$

Now $T(-\Lambda \sqrt{ } t)$ is selfadjoint in the inner product $\left(, \mathrm{G}_{\Lambda}\right)$; it follows, therefore, that $\sup _{\left(\mathbf{x}, \mathbf{G}_{\Lambda} \mathbf{x}\right)=1}\left(T(-\mathbf{\Lambda} \sqrt{ } t) \mathbf{x}, \mathbf{G}_{\Lambda} T(-\mathbf{\Lambda} \sqrt{ } t) \mathbf{x}\right)^{1 / 2}=\max \left\{\left|T\left(-\Lambda_{i} \sqrt{ } t\right)\right|\right\}=T\left(-\Lambda_{\max } \sqrt{ } t\right) \leq 1$ where $\Lambda_{\max }=\max \left\{\Lambda_{i}\right\}$, and thus that

$$
\|T(-\boldsymbol{\Lambda} \sqrt{ } t)\| \leq\left(S_{\mathrm{A}}{ }^{A} S_{A}{ }^{\Lambda}\right){ }^{1 / 2} T\left(-\Lambda_{\max } \sqrt{ } t\right) .
$$

We define $b(t)$ via

$$
\begin{aligned}
b(t) & \equiv \int_{t}^{\infty} p \exp (-p \tau)\|\mathbf{K}\|\left(S_{\Lambda}{ }^{A} S_{A}{ }^{A}\right)^{1 / 2} T\left(-\Lambda_{\max } \sqrt{ } \tau\right) d \tau \\
& =p\|\mathbf{K}\|\left(S_{\Lambda}{ }^{A} S_{A}{ }^{A}\right)^{1 / 2} \frac{\exp \left(-\frac{p t}{2}\right)}{p-\Lambda_{\max }{ }^{2}}\left(T\left(-\Lambda_{\max } \sqrt{ } t\right)+\frac{\Lambda_{\max }}{\sqrt{ } p} T(\sqrt{p t})\right)^{\dagger},
\end{aligned}
$$

where evidently $b(t) \geq\|\mathbf{B}(t)\|$, and find that the asymptotic stability condition, $g<1$, becomes

$$
g=\left(1+\|\mathbf{A}\|\left\|\mathbf{A}^{-1}\right\|\right)\left(S_{\Lambda}{ }^{A} S_{A}{ }^{\Lambda}\right)^{1 / 2}\|\mathbf{K}\| \frac{2-\left(\Lambda_{\max } / \sqrt{ } p\right)}{2 p\left(1-\left(\Lambda_{\max } / \sqrt{ } p\right)\right)^{2}}<1 .
$$

This inequality defines a range of $p$ values over which suspension systems are asymptotically stable, i.e. $\lim _{t \rightarrow \infty} \mathbf{M}(t)=\mathbf{0}$, for admissible operators $\mathbf{D}, \mathbf{K}$ under the further condition that $\boldsymbol{\Lambda}$ and $\mathbf{A}$ be symmetrizable as well as stable.

We can exhibit a second function $b(t)$ and get a second $g$ which is less sharp but more simple. In particular,

$$
T\left(-\Lambda_{\max } \sqrt{ } t\right) \leq 1
$$

therefore we can define $b(t)$ via

$$
\begin{aligned}
b_{2}(t) & \equiv \int_{t}^{\infty} p \exp (-p \tau)\|\mathbf{K}\|\left(S_{\Lambda}{ }^{A} S_{A}{ }^{\Lambda}\right)^{1 / 2} d \tau^{\ddagger} \\
& =\|\mathbf{K}\|\left(S_{\Lambda}^{A} S_{A}^{\Lambda}\right)^{1 / 2} \exp (-p t)
\end{aligned}
$$

where evidently $b_{2}(t) \geq b(t) \geq\|\mathbf{B}(t)\|$. The corresponding $g$ is

$$
g_{2}=\left(1+\|\mathbf{A}\|\left\|\mathbf{A}^{-1}\right\|\right)\left(S_{\Lambda}{ }^{A} S_{\mathbf{A}}{ }^{\Lambda}\right)^{1 / 2}\|\mathbf{K}\| \frac{1}{p}
$$

If $\mathbf{G} \in \mathcal{G}_{-D} \cap g_{K} \neq \varnothing$, we can calculate $p_{0}$ such that for $p>p_{0} \lim _{t \rightarrow \infty} \mathbf{M}(t)=\mathbf{0}$. Now $\mathbf{G} \in G_{\Lambda}$ and $\mathbf{G D}^{1 / 2},-\mathrm{GK} \in \mathrm{G}_{\Lambda}$; therefore if

$$
\mathrm{G}_{A}=\mathrm{G}, \quad \mathrm{G}_{\Lambda}=\mathrm{GD}^{-1 / 2} \text { then } S_{\Lambda}{ }^{A} S_{A}{ }^{\Lambda}=\frac{D_{\max }{ }^{1 / 2}}{D_{\min }{ }^{1 / 2}}
$$

whereas if

$$
\mathrm{G}_{A}=\mathrm{G}, \quad \mathrm{G}_{\mathrm{A}}=-\mathrm{GK} \text { then } \quad S_{\mathrm{A}}{ }^{A} S_{A}{ }^{A}=\frac{K_{\min }}{K_{\max }}
$$

$\dagger$ It is not necessary that $\mathbf{A}$ be symmetrizable; $\mathbf{A}$ normalizable is sufficient.

$\ddagger$ If $\boldsymbol{\Lambda}$ is normalizable and stable, $b_{2}(t)$ is the best bound of $\|\mathbf{B}(t)\|$ that can be produced in $\mathbf{a}$ general analysis. 
further because $\mathbf{G} \in \mathcal{G}_{-D} \cap \mathcal{G}_{K}$

$$
\left\|\mathrm{A}^{-1}\right\|\|\mathbf{A}\| \leq \frac{K_{\max }{ }^{-1}-p^{-1 / 2} D_{\min }^{-1 / 2}}{K_{\min }{ }^{-1}-p^{-1 / 2} D_{\max }{ }^{-1 / 2}} \leq \max \left\{\frac{K_{\min }}{K_{\max }}, \frac{D_{\max }{ }^{1 / 2}}{D_{\min }{ }^{1 / 2}}\right\}
$$

and

$$
\|\mathbf{K}\|=-K_{\min } .
$$

It follows that

$$
g_{2} \leq-K_{\min }\left(1+\frac{K_{\max }^{-1}-p^{-1 / 2} D_{\min }^{-1 / 2}}{K_{\min }{ }^{-1}-p^{-1 / 2} D_{\max }{ }^{-1 / 2}}\right)\left(S_{A}{ }^{A} S_{A}{ }^{\Lambda}\right)^{1 / 2} \frac{1}{p}
$$

The right-hand side of (6) is less than 1 for all $p>p_{0}$ where $p_{0}{ }^{1 / 2}$, the largest positive zero of

$$
\begin{aligned}
-K_{\min }{ }^{-1} z^{3}+D_{\max }{ }^{-1 / 2} z^{2}-\left(S_{\Lambda}{ }^{A} S_{A}{ }^{\Lambda}\right)^{1 / 2}(1+ & \left.\frac{K_{\min }}{K_{\max }}\right) z \\
& +\left(S_{\Lambda}{ }^{A} S_{A}{ }^{\Lambda}\right)^{1 / 2} K_{\min }\left(D_{\max }{ }^{-1 / 2}+D_{\min }{ }^{-1 / 2}\right),
\end{aligned}
$$

is bounded above by $p_{1}{ }^{1 / 2}$,

$$
p_{1}=-K_{\min }\left(S_{\Lambda}^{A} S_{A}{ }^{A}\right)^{1 / 2}\left(1+\max \left\{\frac{K_{\min }}{K_{\max }}, \frac{D_{\max }{ }^{1 / 2}}{D_{\min }{ }^{1 / 2}}\right\}\right) .
$$

Thus for all $p>p_{1}$ we find that $\mathbf{M}(t) \rightarrow \mathbf{0}$.

4. Asymptotic stability of Eq. (3) under the condition $\mathcal{G}_{-n} \cap g_{K} \neq \varnothing$. The stability condition $p>p_{0}$ of the foregoing section can be sharpened in the special case of thermodynamically ideal solutions. Such a limitation is physically realizable and provides physicochemical parameters, $\mathbf{D}$ and $\mathbf{K}$, for which the films bounding the particles are well behaved and for which suspensions of well-behaved particles should themselves be well behaved. The ideal solutions belong to a larger class of systems, investigated hereinafter, for which $\mathcal{G}_{-D} \cap \mathcal{G}_{K} \neq \varnothing$ and for which the question of the location of the latent roots of the $\zeta$-matrix, $\mathbf{f}(\zeta)$, may be addressed directly. Thus we let $\mathbf{G} \in \mathcal{G}_{-D} \cap \mathrm{G}_{K}$ and investigate the latent roots of $\mathbf{f}(\zeta) \mathbf{K}^{-1}=\left(\zeta^{2}-p\right) \zeta\left(\zeta \mathbf{K}^{-1}-\mathbf{D}^{-1 / 2}\right)-p \mathbf{I}$, using a generalization of a method of Duffin [6] which gives information about the latent roots of a $\zeta$-matrix $g(\zeta ; \mathbf{U}, \mathbf{V}, \cdots)$ whenever certain information about its scalar counterpart $g(\zeta ; u, v, \cdots)$ is available.

In what follows we must allow for $\operatorname{im}\left(\zeta_{i}\right) \neq 0$; thus we let $\zeta_{i} \in C, \mathbf{x}_{i} \in C^{r}$ be a latent root and a corresponding latent vector of $\mathbf{f}(\zeta) \mathbf{K}^{-1}$, where $\left(\mathbf{x}_{i}, \mathbf{G x}_{i}\right)=1$. Thus $\mathbf{f}\left(\zeta_{i}\right) \mathbf{K}^{-1} \mathbf{x}_{i}=\mathbf{0}$ so that

$$
\left(-\mathbf{f}\left(\zeta_{i}\right) \mathbf{K}^{-1} \mathbf{x}_{i}, \mathbf{G} \mathbf{x}_{i}\right)=\left(\zeta_{i}{ }^{2}-p\right) \zeta_{i}\left(\zeta_{i}\left(-\mathbf{K}^{-1} \mathbf{x}_{i}, \mathbf{G} \mathbf{x}_{i}\right)+\left(\mathbf{D}^{-1 / 2} \mathbf{x}_{i}, \mathbf{G x}_{i}\right)\right)+p=0
$$

and hence $\zeta_{i} \in \cup Z(\mathbf{x})$ where

$$
Z(\mathbf{x})=\left\{z:\left(z^{2}-p\right) z\left(z\left(-\mathbf{K}^{-1} \mathbf{x}, \mathbf{G} \mathbf{x}\right)+\left(\mathbf{D}^{-1 / 2} \mathbf{x}, \mathbf{G} \mathbf{x}\right)\right)+p=0,(\mathbf{x}, \mathbf{G x})=1\right\} .
$$

Eq. (3) is asymptotically stable iff $\operatorname{re}\left(\zeta_{i}\right)<0$ or $\operatorname{re}\left(\zeta_{i}{ }^{2}\right)<p$ if $\operatorname{re}\left(\zeta_{i}\right) \geq 0$; thus we seek conditions under which $\operatorname{re}\left(z^{2}\right)<p$ whenever $\operatorname{re}(z) \geq 0$ and $z \in \cup Z(\mathbf{x})$.

Because $\mathbf{G} \in \mathcal{G}_{-D} \cap \mathcal{S}_{K}$, we conclude, $\forall \mathbf{x} \in C^{r}$, that $\left(\mathbf{K}^{-1} \mathbf{x}, \mathbf{G x}\right)$ and $\left(\mathbf{D}^{-1 / 2} \mathbf{x}, \mathbf{G} \mathbf{x}\right)$ 
are real and further, if $(\mathbf{x}, \mathbf{G x})=1$, that

$$
0<-K_{\min }^{-1} \leq\left(-\mathbf{K}^{-1} \mathbf{x}, \mathbf{G x}\right) \leq-K_{\max }^{-1}
$$

and

$$
0<D_{\max }^{-1 / 2} \leq\left(\mathrm{D}^{-1 / 2} \mathbf{x}, \mathrm{Gx}\right) \leq D_{\min }^{-1 / 2}
$$

where $K_{\min }=\min \left\{K_{i}\right\}$, etc. We let

$$
\begin{aligned}
\boldsymbol{z} & \equiv z\left(-\mathbf{K}^{-1} \mathbf{x}, \mathbf{G x}\right)^{1 / 2} \\
\alpha & \equiv p\left(-\mathbf{K}^{-1} \mathbf{x}, \mathbf{G} \mathbf{x}\right)>0, \\
\beta & \equiv\left(-\mathbf{K}^{-1} \mathbf{x}, \mathbf{G x}\right)^{-1 / 2}\left(\mathbf{D}^{-1 / 2} \mathbf{x}, \mathbf{G x}\right)>0,
\end{aligned}
$$

where, for $(\mathbf{x}, \mathbf{G x})=1, \alpha \in\left[\alpha_{\min }, \alpha_{\max }\right], \beta \in\left[\beta_{\min }, \beta_{\max }\right]$ and where $\alpha_{\min }=-K_{\min }{ }^{-1} p$, $\beta_{\min }=D_{\max }^{-1 / 2}\left(-K_{\max }\right)^{1 / 2}$, etc.

We observe that $z \in Z(\mathbf{x})$ iff $z \in Z(x)=\left\{z: P(z ; \alpha, \beta) \equiv\left(z^{2}-\alpha\right)\right\}(z+\beta)+\alpha=0$; $(\mathbf{x}, \mathbf{G x})=1\}$; further, $\operatorname{re}\left(z^{2}\right)<p$ whenever $\operatorname{re}(z)>0$ and $z \in \cup Z(\mathbf{x})$ iff $\operatorname{re}\left(z^{2}\right)<\alpha$ whenever re $(z)>0$ and $z \in \cup Z(x)$. It follows that conditions under which $\operatorname{re}\left(z^{2}\right)<\alpha$ if $\operatorname{re}(z)>0$ and $P(z ; \alpha, \beta)=0 \forall(\alpha, \beta) \in\left[\alpha_{\min }, \alpha_{\max }\right] \times\left[\beta_{\min }, \beta_{\max }\right]$ are sufficient for asymptotic stability of Eq. (3) inasmuch as $\cup 3(\mathbf{x}) \subset\{z: P(z ; \alpha, \beta)=0,(\alpha, \beta) \in$ $\left.\left[\alpha_{\min }, \alpha_{\max }\right] \times\left[\beta_{\min }, \beta_{\max }\right]\right\}$. Thus for given values of $p, \mathbf{D}, \mathbf{K}$ we have reduced the investigation of the $4 r$ zeros $\operatorname{det}(f(\zeta))$ to the investigation of the 4 zeros $P(z ; \alpha, \beta)$ wherein the coefficients $\alpha, \beta$ are indeterminate and range over a rectangle of the $\alpha-\beta$ plane.

Now $P(z ; \alpha, \beta)$ arises naturally if $r=1$. In that context the values of $\alpha$ and $\beta$ are determinate, given $p, \mathbf{D}, \mathbf{K}$, and a region of the $\alpha-\beta$ plane can be identified wherein $\operatorname{re}\left(z^{2}\right)<\alpha$ if $\operatorname{re}(\xi)>0$ and $P(\xi ; \alpha, \beta)=0$.

This information on $P(z ; \alpha, \beta)$ is summarized in Fig. 1 and is given in detail by DeGance [3]. It follows that a sufficient condition for the asymptotic stability of Eq. (3), given $p, \mathbf{D}$ and $\mathbf{K}$, subject to $\mathcal{G}_{-D} \cap \varrho_{K} \neq \varnothing$, is that the rectangle of allowable $(\alpha, \beta)$ lie within the shaded region of Fig. 1 which is the proven region of asymptotic stability for parametric $p, \mathbf{D}, \mathbf{K}$ in the case $r=1$.

If for $r=1$ we conjecture asymptotic stability over the entire positive quadrant of the $\alpha-\beta$ plane, we conclude, for all $r$, asvmptotic stability for all $p, \mathbf{D}, \mathbf{K}$ such that $S_{-D} \cap \mathcal{G}_{K} \neq \varnothing$.

5. Positivity. We let $\mathbf{x} \in R^{N}$, admit singular operators to the class of physically allowable $\mathbf{D}, \mathbf{K} \in L\left(R^{N}\right)$ and reexamine the question of physicochemical stability.

We say that $\mathbf{x}$ is positive, $\mathbf{x} \geq 0$, iff $x_{i} \geq 0, i=1, \cdots, N$; further $\mathbf{x}$ is a physically allowable state vector iff $\mathbf{x} \geq 0$ and $\sum_{i=1}{ }^{N} x_{i}=1$. Insofar as $\mathbf{x}$ is physically allowable, it is bounded and constraints arise on the operators $\mathbf{D}$ and $\mathbf{K}$ descriptive of particulate and/or suspension processes. Evidently such constraints are related to stability; in particular, we give the constraints on $\mathbf{D}^{-1 / 2} \mathrm{~K}$ that arise in connection with the stability of a catalyst particle plunged into an initially uniform reservoir of infinite extent, viz. Eq. (2).

We investigate a class of problems related to the vector renewal equation; in particular, we observe that if $\mathbf{x}(t)=\mathbf{M}(t) \mathbf{x}(0)$, where $\mathbf{x}(0) \geq 0$, then $\mathbf{x}(t) \geq 0, \sum_{i=1}{ }^{N} x_{i}(t)=$ $\sum_{i=1}^{N} x_{i}(0)$ iff $\mathbf{M}(t) \in M$. We obtain conclusions which generalize the classic theorem, $\exp (\mathrm{V} t) \in M$ iff $\mathrm{V} \in \Phi_{0}$, and its extension, $\exp (\mathrm{V} t) \in P$ iff $\mathrm{V} \in \Phi$ (Bellman [1]). 


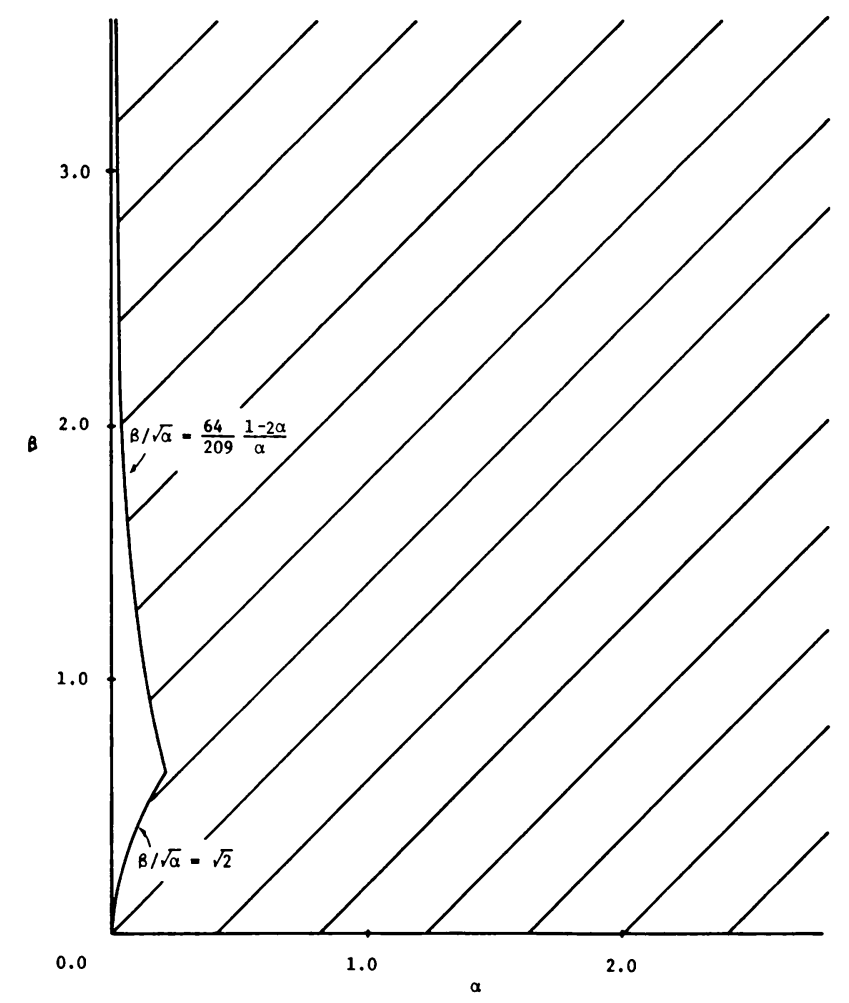

FIG. 1. Proven region of asymptotic stability for $r=1$.

We make use of the following lemma:

Lemma B. Let $\mathbf{F}(t)$ and $\mathbf{U}(t) \in P, \mathbf{F} \in C[0, T], \mathbf{U} \in \mathfrak{R}[0, T]$ and let $\mathbf{X}$ satisfy

$$
\mathbf{X}(t)=\mathbf{F}(t)+\int_{0}^{t} \mathbf{U}(t-\tau) \mathbf{X}(\tau) d \tau, \quad t \in[0, T] ;
$$

then $\mathbf{X}(t) \in P, \mathbf{X} \in C[0, T]$.

The proof follows on construction of $\mathbf{X}$ (cf. Bellman and Cooke [2] for the proof of the scalar version of Lemma B).

We would like to have the conclusions of Lemma B for kernel operators $\mathbf{U}(t)$ not limited to $P$. In particular, $\mathbf{U}(t) \in \Phi_{0}$ is more expressive of elementary chemical relations than is $\mathbf{U}(t) \in P$; thus, for $\mathbf{U}(t) \in \Phi_{0}$, where $\Phi_{0}, P \subset \Phi, \Phi_{0} \cap P=\varnothing$, what follows can be interpreted as a generalization of Lemma $B$, under certain limitations on the $t$-dependence of $\mathrm{U}$.

The integral equations descriptive of the systems introduced in Sec. 1 take the form

$$
\mathbf{x}(t)=\mathbf{x}(0)+\int_{0}^{t} k(\mathbf{U}, t-\tau) \mathbf{V} \mathbf{x}(\tau) d \tau
$$

hereinafter we investigate the special case

$$
\mathbf{x}(t)=\mathbf{x}(0)+\int_{0}^{t} k(t-\tau) \mathbf{V} \mathbf{x}(\tau) d \tau .
$$


Now in the eigenbasis of $\mathrm{V}$, Eq. (7) reduces to $N$ scalar equations for the coefficients of $\mathbf{x}$; however, the condition $\mathbf{x} \geq 0$ is not readily translated into conditions on the coefficients of $\mathbf{x}$ in a basis other than $\left\{\mathbf{e}_{i}\right\}$. Thus we cannot make direct use of the scalar version of Lemma $\mathbf{B}$, and we write $\mathbf{x}(t)=\mathbf{M}(t) \mathbf{x}(0)$ where

$$
\mathbf{M}(t)=\mathbf{I}+\int_{0}^{t} k(t-\mathbf{x}) \mathbf{V} \mathbf{M}(\tau) d \tau .
$$

We investigate Eq. (8) under the condition that $k(t)>0, t \in(0, T]$ and prove first that if $\mathbf{M}(t) \in M$ then $\mathbf{V} \in \Phi_{0}$. The generalization that if $\mathbf{M}(t) \in P$ then $\mathbf{V} \in \Phi$ is immediate.

If $\mathbf{M}(t) \in M$, then

$$
\mathbf{u}^{+} \mathbf{M}(t)=\mathbf{u}^{+}, \mathbf{u}=\left(\begin{array}{c}
1 \\
\vdots \\
1
\end{array}\right)
$$

It follows from Eq. (8) that

$$
\int_{0}^{t} k(t-\tau) \mathbf{u}^{+} \mathbf{V M}(\tau) d \tau=0^{+}, \quad t \in[0, T]
$$

and thus that

$$
\mathbf{u}^{+} \mathbf{V M}(t)=\mathbf{0}^{+}, \quad t \in[0, T]
$$

inasmuch as $k(t)>0, t \in(0, T]$. Now $\mathbf{M}(0)=\mathbf{I}$; hence $\mathbf{M}(t)$ is nonsingular for $t \in[0, \epsilon]$; thus $\mathbf{u}^{+} \mathbf{V}=\mathbf{0}^{+}$so that if $\mathbf{V} \in \Phi$ then $\mathbf{V} \in \Phi_{0}$.

We let $\|\mathbf{V}\|=\sum_{i=1}{ }^{N} \sum_{i=1}{ }^{N}\left|v_{i i}\right|$, observe that $\|\mathbf{U V}\| \leq\|\mathbf{U}\|\|\mathbf{V}\|$ (cf. Bellman [1]), and construct $\mathbf{M}(t)$ in the neighborhood of $t=0$. We choose $T$ so that

$$
\left\|\int_{0}^{T} k(\tau) \mathbf{V} d \tau\right\|=\int_{0}^{T} k(\tau) d \tau\|\mathbf{V}\|=1
$$

and observe that

$$
\epsilon(t) \equiv\left\|\int_{0}^{t} k(\tau) \mathbf{V} d \tau\right\|<1, \quad t \in[0, T) .
$$

Thus the sequence of Picard iterates of Eq. (8) converges uniformly to $\mathbf{M}(t)$ on every subinterval of $[0, T)$, i.e.

$$
\mathbf{M}(t)=\mathbf{I}+\int_{0}^{t} k(\tau) d \tau \mathbf{V}+\int_{0}^{t} k(t-\tau) \int_{0}^{\tau} k\left(\tau_{0}\right) d \tau_{0} d \tau \mathbf{V}^{2}+\cdots
$$

where convergence is uniform on $[0, T)$, and

$$
\left\|\mathbf{M}(t)-\left(\mathbf{I}+\int_{0}^{t} k(\tau) d \tau \mathbf{V}\right)\right\| \leq \frac{\epsilon^{2}}{1-\epsilon}, \quad t \in[0, T)
$$

(cf. Miller [11]).

If we suppose that at least one non-diagonal element of $\mathrm{V}$ is negative, viz. $v_{i i}=$ $-\delta<0, i \neq j$, then it follows that 


$$
\begin{aligned}
0<m_{i j}(t)+\delta \int_{0}^{t} k(\tau) d \tau & =\left|m_{i j}(t)-\int_{0}^{t} k(\tau) d \tau v_{i i}\right| \leq\left\|\mathbf{M}(t)-\left(\mathbf{I}+\int_{0}^{t} k(\tau) d \tau \mathbf{V}\right)\right\| \\
& \leq \frac{\epsilon^{2}}{1-\epsilon} .
\end{aligned}
$$

Thus we conclude that

$$
0<\delta \int_{0}^{t} k(\tau) d \tau \leq \frac{\epsilon^{2}}{1-\epsilon}, \quad t \in(0, T)
$$

and thus, on using the definition of $\epsilon$, that

$$
0<\delta \leq \frac{\int_{0}^{t} k(\tau) d \tau\|\mathbf{V}\|^{2}}{1-\int_{0}^{t} k(\tau) d \tau\|\mathbf{V}\|}, \quad t \in[0, T)
$$

which is a contradiction for $t \rightarrow 0$. Thus the supposition that a nondiagonal element of $\mathbf{V}$ is negative is false and we conclude that if $\mathbf{M}(t) \in M$ then $\mathbf{V} \in \Phi$ and hence $\mathbf{V} \in \Phi_{0}$.

We now give a limited converse. In particular, we let $\mathbf{V} \in \Phi_{0}$ and identify a class of positive functions $k \in \mathfrak{R}[0, T], T<\infty$, such that $\mathbf{M}(t) \in M, t \geq 0$.

We let

$$
m(t ; k, v)=1+\int_{0}^{t} k(t-\tau) v m(\tau ; k, v) d \tau
$$

and observe that $\mathbf{M}(t)=m(t ; k, \mathbf{V})$, viz.,

$$
m(t ; k, \mathbf{V})=\mathbf{I}+\int_{0}^{t} k(t-\tau) \mathbf{V} m(\tau ; k, \mathbf{V}) d \tau
$$

where $m(t ; k, v)$, and thus $m(t ; k, \mathrm{~V})$, exists, is unique and its Laplace transformation exists. Thus on letting $\mathfrak{m}(s ; k, \mathrm{~V})$ be the Laplace transformation of $m(t ; k, \mathrm{~V})$, etc., we find that

$$
\mathfrak{m}(s ; k, \mathbf{V})=s^{-1} \mathbf{I}+\mathfrak{t}(s) \mathbf{V} \mathfrak{m}(s ; k, \mathbf{V})
$$

which, on using $\mathbf{V}=(\mathbf{V}-\operatorname{diag}(\mathbf{V}))+\operatorname{diag}(\mathbf{V})$, where $\operatorname{diag}(\mathbf{V})=\operatorname{diag}\left(v_{11}, \cdots, v_{N N}\right)$, becomes

$$
\mathfrak{m}(s ; k, \mathbf{V})=\operatorname{diag}^{-1}(s(\mathbf{I}-\mathfrak{f}(s) \mathbf{V}))+\operatorname{diag}^{-1}\left(\frac{\mathbf{I}-\mathfrak{f}(s) \mathbf{V}}{\mathfrak{f}(s)}\right)(\mathbf{V}-\operatorname{diag}(\mathbf{V})) \mathfrak{m}(s ; k, \mathbf{V}) .
$$

We now let

$$
\mathfrak{r}(s ; k, v)=\mathfrak{f}(s)(1-\mathfrak{f}(s) v)^{-1}
$$

and observe that

$$
r(t ; k, v)=k(t)+\int_{0}^{t} k(t-\tau) v r(\tau ; k, v) d \tau
$$

thus, on using $\mathfrak{m}\left(s ; v_{i i}, k\right)=s^{-1}\left(1-\mathfrak{f}(s) v_{i i}\right)^{-1}$, we find, on calculating the inverse Laplace transformation of Eq. (8), that

$$
m(t ; k, \mathbf{V})=m(t ; k, \operatorname{diag}(\mathbf{V}))+\int_{0}^{t} r(\tau ; k, \operatorname{diag}(\mathbf{V}))(\mathbf{V}-\operatorname{diag}(\mathbf{V})) m(t-\tau ; k, \mathbf{V}) d \tau
$$


If $\mathbf{V} \in \Phi$ it follows that $\mathbf{V}-\operatorname{diag}(\mathbf{V}) \in P$; thus we conclude, using Lemma $\mathbf{B}$, that if $\mathrm{V} \in \Phi$ and if $m(t ; k, v) \geq 0, r(t ; k, v) \geq 0$, then $\mathbf{M}(t)=m(t ; k, \mathbf{V}) \in P$. Further, if $k \in C^{(1)}[0, T]$ then

$$
r(t ; k, v)=m(t ; k, v) k(0)+\int_{0}^{t} m(t-\tau ; k, v) k^{(1)}(\tau) d \tau
$$

it follows, therefore, that $r(t ; k, v) \geq 0$ if $m(t ; k, v) \geq 0$ and if $k^{(1)}(t) \geq 0, k(0) \geq 0$.

Thus if $k \geq 0 \in C^{(1)}[0, T]$ and if $m(t ; k, v) \geq 0$ and $k^{(1)}(t) \geq 0$ we can conclude that if $\mathbf{V} \in \Phi$ then $\mathbf{M}(t)=m(t ; k, \mathbf{V}) \in P, t \in[0, T]$. It follows immediately that if $\mathbf{V} \in \Phi_{0}$ then $\mathbf{M}(t) \in M$. Finally we observe that $m(t ; k, \mathbf{V})-m(t ; k, \operatorname{diag}(\mathbf{V})) \in P$, $t \in[0, T]$, cf. Eq. (8).

We draw two conclusions from the foregoing that are important in spatially nonuniform chemically active systems, viz., a planar catalyst surface plunged into an initially uniform reservoir of infinite extent, (cf. Eq. (2)). We show that $T(-\mathrm{V} \sqrt{ } t) \in M$ iff $\mathbf{V} \in \Phi_{0}$ and the corollary that erfc $(-\mathrm{V} \sqrt{ } t) \in M$ if $\mathbf{V},-\mathbf{V}^{2} \in \Phi_{0}$.

We let $\mathbf{M}(t)=m(t ; k, \mathbf{V})=T(-\mathbf{V} \sqrt{ } t)$, where $T(x)=\exp \left(x^{2}\right) \operatorname{erfc}(x) \geq 0$, $x \in(-\infty, \infty)$, and observe that

$$
T(-\mathrm{V} \sqrt{ } t)=\mathrm{I}+\int_{0}^{t} \frac{1}{\sqrt{ } \pi(t-\tau)^{1 / 2}} \mathrm{~V} T(-\mathrm{V} \sqrt{ } \tau) d \tau
$$

and therefore, that

$$
r\left(t ; \frac{1}{(\pi t)^{1 / 2}}, v\right)=\frac{1}{(\pi t)^{1 / 2}}+v T(-v \sqrt{ } t) .
$$

It follows that if $r\left(t ; 1 /(\pi t)^{1 / 2}, v\right) \geq 0, v \in(-\infty, 0)$ then $T(-\mathrm{V} \sqrt{ } t) \in M$ iff $\mathrm{V} \in \Phi_{0}$. On using Eq. (7.1.13) of [7] we find that

$$
T(-v \sqrt{ } t) \leq \frac{2}{\left(\pi v^{2} t\right)^{1 / 2}+\left(\pi v^{2} t+4\right)^{1 / 2}}
$$

and therefore that

$$
\frac{-1}{(\pi t)^{1 / 2}}<v T(-v \sqrt{ } t)<0
$$

from which it follows that $r\left(t ; 1 /(\pi t)^{1 / 2}, v\right) \geq 0$. Further if $\mathbf{V},-\mathbf{V}^{2} \in \Phi_{0}$ then erfc $(-\mathbf{V} \sqrt{ } t)$ $\in M$ because erfc $(-\mathrm{V} \vee t)=\exp \left(-\mathrm{V}^{2} t\right) T(-\mathrm{V} \vee t), \exp \left(-\mathrm{V}^{2} t\right) \in M$ iff $-\mathrm{V}^{2} \in \Phi_{0}$ and a product of Markoff factors is itself Markoff.

Now the solution of Eq. (2) is

$$
\mathbf{y}(t)=T\left(-\mathbf{D}^{-1 / 2} \mathbf{K} \sqrt{ } t\right) \mathbf{y}(0)
$$

and for systems so represented the minimal condition of physical faithfulness is that $T\left(-\mathrm{D}^{-1 / 2} \mathrm{~K} \vee t\right) \in M, t \in[0, \infty)$. We conclude, therefore, that physically allowable $\{D, K\}$ must satisfy the condition $D^{-1 / 2} \mathbf{K} \in \Phi_{0}$ in addition to whatever conditions must be placed on $\mathbf{D}$ and $\mathbf{K}$ separately, principally $\mathbf{K} \in \Phi_{0}$, but also $\mathcal{G}_{-D}{ }^{0}, \mathcal{S}_{K}{ }^{0}$ nonempty where

$$
{\mathcal{G}_{-D}^{0}}^{0}=\left\{\mathrm{G}=\mathrm{G}^{+}>0: \mathrm{GD}=(\mathrm{GD})^{+} \geq 0\right\} .
$$

We remark firstly that the condition $\mathbf{D}^{-1 / 2} \mathbf{K} \in \Phi_{0}$ is a stability condition which 
implies $\left|\arg \left(\Lambda_{i}\right)\right| \geq \frac{1}{2} \pi$; it complements the stability condition $\left|\arg \left(\Lambda_{i}\right)\right|>\frac{1}{4} \pi$ which follows from Eq. (2) on imposing the condition that $\|\mathbf{y}(t)\|$ remain bounded for $t \in[0, \infty)$.

We remark secondly that insofar as Eq. (2) arises on linearization of a nonlinear theory, we may not rightly presume $T\left(-\mathrm{D}^{-1 / 2} \mathrm{~K} \sqrt{ } t\right) \in M$ if the linearization is to account for small excursions from a reference state far from the boundary of state space; there we may presume only $\mathbf{u}^{+} T\left(-\mathrm{D}^{-1 / 2} \mathbf{K} \sqrt{ } t\right)=\mathbf{u}^{+}$. Nonetheless all of the foregoing is pertinent for small excursions from reference states on or near the boundary of the state space, i.e. on or near the hyperplanes $x_{i}=0, i=1, \cdots, N$, and places conditions on the operators arising therein.

\section{REFERENCES}

[1] R. Bellman, Introduction to matrix analysis, McGraw-Hill, New York, 1970

[2] R. Bellman and K. L. Cooke, Differential-difference equations, Academic Press, New York, 1963

[3] A. E. DeGance, Diffusion-heterogeneous chemical reaction interference in multicomponent systems, Ph.D. dissertation, University of Florida, 1974

[4] A. E. DeGance and L. E. Johns, The sensitivity of the selectivity to transport parameters in batch heterogeneous chemical reaction systems, Chem. Eng. J. 7, 227-244 (1974)

[5] R. Driver, Some harmless delays, pp. 103-119 in Delay and functional differential equations and their applications (ed. K. Schmitt), Academic Press, New York, 1972

[6] R. J. Duffin, A minimax theory for overdamped networks, J. Rat. Mech. and Analysis 4, 221-233 (1955)

[7] Handbook of mathematical functions, N. B. S. AMS 55, Government Printing Office, Wàshington, D. C., 1965

[8] J. L. Hudson, Transient multicomponent diffusion with heterogeneous reaction, A.I.Ch.E. J. 13, 961-964 (1967)

[9] L. E. Johns and X. B. Reed, Diffusion-heterogeneous chemical reaction in multicomponent systems. I. The penetration theory for single catalyst particles in a dilute turbulent suspension, Chem. Engng. Sci. 28, 275-288 (1973)

[10] L. E. Johns and X. B. Reed, On imperfect contacting in batch heterogeneous chemical reaction systems, Chem. Engng. Sci. 28, 1591-1609 (1973)

[11] R. K. Miller, Nonlinear Volterra integral equations, Benjamin, Menlo Park, 1971

[12] J. A. Nohel, Some problems in nonlinear Volterra integral equations, Bull. Amer. Math. Soc. 68, $323-329$ (1962)

[13] G. E. Shilov, An introduction to the theory of linear spaces, Prentice-Hall, Englewood Cliffs, 1961 